\title{
Observational tests of interstellar methanol formation
}

\author{
E. S. Wirström ${ }^{1,2}$, W. D. Geppert ${ }^{3}$, Å. Hjalmarson², C. M. Persson² , J. H. Black² , P. Bergman² , T. J. Millar ${ }^{4}$, \\ M. Hamberg ${ }^{3}$, and E. Vigren ${ }^{3}$ \\ 1 Astrochemistry Laboratory and The Goddard Center for Astrobiology, Mailstop 691, NASA Goddard Space Flight Center, \\ Greenbelt, MD 20770, USA \\ e-mail: eva.s.wirstrom@nasa.gov \\ 2 Onsala Space Observatory, Department of Earth and Space Sciences, Chalmers University of Technology, 43992 Onsala, Sweden \\ 3 Physics Department, Stockholm University, 10691 Stockholm, Sweden \\ 4 Astrophysics Research Centre, School of Mathematics and Physics, Queen's University Belfast, Belfast BT7 1NN, UK
}

Received 16 January 2011 / Accepted 14 July 2011

\begin{abstract}
Context. It has been established that the classical gas-phase production of interstellar methanol $\left(\mathrm{CH}_{3} \mathrm{OH}\right)$ cannot explain observed abundances. Instead it is now generally thought that the main formation path has to be by successive hydrogenation of solid $\mathrm{CO}$ on interstellar grain surfaces.

Aims. While theoretical models and laboratory experiments show that methanol is efficiently formed from CO on cold grains, our aim is to test this scenario by astronomical observations of gas associated with young stellar objects (YSOs).

Methods. We have observed the rotational transition quartets $J=2_{K}-1_{K}$ of ${ }^{12} \mathrm{CH}_{3} \mathrm{OH}$ and ${ }^{13} \mathrm{CH}_{3} \mathrm{OH}$ at 96.7 and $94.4 \mathrm{GHz}$, respectively, towards a sample of massive YSOs in different stages of evolution. In addition, the $J=1-0$ transitions of ${ }^{12} \mathrm{C}^{18} \mathrm{O}$ and ${ }^{13} \mathrm{C}^{18} \mathrm{O}$ were observed towards some of these sources. We use the ${ }^{12} \mathrm{C} /{ }^{13} \mathrm{C}$ ratio to discriminate between gas-phase and grain surface origin: If methanol is formed from $\mathrm{CO}$ on grains, the ratios should be similar in $\mathrm{CH}_{3} \mathrm{OH}$ and $\mathrm{CO}$. If not, the ratio should be higher in $\mathrm{CH}_{3} \mathrm{OH}$ due to ${ }^{13} \mathrm{C}$ fractionation in cold $\mathrm{CO}$ gas. We also estimate the abundance ratios between the nuclear spin types of methanol (E and $\mathrm{A}$ ). If methanol is formed on grains, this ratio is likely to have been thermalized at the low physical temperature of the grain, and therefore show a relative over-abundance of A-methanol.

Results. We show that the ${ }^{12} \mathrm{C} /{ }^{13} \mathrm{C}$ isotopic ratio is very similar in gas-phase $\mathrm{CH}_{3} \mathrm{OH}$ and $\mathrm{C}^{18} \mathrm{O}$, on the spatial scale of about $40^{\prime \prime}$, towards four YSOs. For two of our sources we find an overabundance of A-methanol as compared to E-methanol, corresponding to nuclear spin temperatures of 10 and $16 \mathrm{~K}$. For the remaining five sources, the methanol E/A ratio is less than unity.

Conclusions. While the ${ }^{12} \mathrm{C} /{ }^{13} \mathrm{C}$ ratio test is consistent with methanol formation from hydrogenation of $\mathrm{CO}$ on grain surfaces, the result of the $\mathrm{E} / \mathrm{A}$ ratio test is inconclusive.
\end{abstract}

Key words. ISM: molecules - astrochemistry - radio lines: ISM

\section{Introduction}

Gas phase methanol is readily observed in a wide range of interstellar environments, with typical abundances relative to $\mathrm{H}_{2}$ of $10^{-6}-10^{-7}$ in hot cores, $10^{-9}$ in dark clouds, and $<10^{-9}$ in diffuse molecular gas (Liszt et al. 2008). In classical ion-neutral chemistry it is mainly produced by a two-step mechanism from methyl ions (which in turn are formed from $\mathrm{C}^{+}$),

$$
\begin{aligned}
\mathrm{CH}_{3}^{+}+\mathrm{H}_{2} \mathrm{O} & \longrightarrow \mathrm{CH}_{3} \mathrm{OH}_{2}^{+} \\
\mathrm{CH}_{3} \mathrm{OH}_{2}^{+}+\mathrm{e}^{-} & \longrightarrow \mathrm{CH}_{3} \mathrm{OH}+\mathrm{H} .
\end{aligned}
$$

However, it has been established that the dissociative recombination of $\mathrm{CH}_{3} \mathrm{OH}_{2}^{+}$in the second step cannot be efficient enough for this gas-phase production to alone explain the observed abundances (Geppert et al. 2006; Garrod et al. 2006).

Interstellar methanol is also observed in its solid form towards high- and low-mass protostars, at abundances of a few to a few tens of percent relative to water ice (Allamandola et al. 1992; Brooke et al. 1999; Pontoppidan et al. 2003; Bottinelli et al. 2010), sometimes being the second most abundant ice observed (Dartois et al. 1999). The observed presence of interstellar methanol ice supports the now favoured formation path, which is successive hydrogenation of solid $\mathrm{CO}$ on cold grains, resulting in methanol and, at an intermediate stage, formaldehyde. Experimentally, this process has been shown to be effective (Watanabe et al. 2004; Fuchs et al. 2009), and methanol abundances observed in translucent clouds are well reproduced by models including $\mathrm{CO}$ hydrogenation on grains, while pure gas-phase models fail by 4 orders of magnitude (Turner 1998).

As a complement to chemical models and laboratory experiments, and in order to establish firmly whether methanol is formed from $\mathrm{CO}$ on grains or not, we need some observational tests. One such test, isotope labelling "a posteriori", was suggested by Charnley et al. (2004). It is based on the observed effect of ${ }^{13} \mathrm{C}$ fractionation into $\mathrm{CO}$ at low temperatures due to the reaction

${ }^{13} \mathrm{C}^{+}+{ }^{12} \mathrm{CO} \longrightarrow{ }^{13} \mathrm{CO}+{ }^{12} \mathrm{C}^{+}+\Delta E$,

where $\Delta E=35 \mathrm{~K}$. This results in an overabundance of ${ }^{13} \mathrm{CO}$, and a ${ }^{13} \mathrm{C}$ deficiency in other molecules forming from ion-neutral reactions involving $\mathrm{C}^{+}$in the gas-phase (Langer et al. 1984). If this selective fractionation remains unaltered by the processes of adsorption and desorption, then the ${ }^{12} \mathrm{C} /{ }^{13} \mathrm{C}$ ratio in various molecules could be used to distinguish between formation from $\mathrm{CO}$ on cold grains and gas-phase formation. Therefore Charnley et al. (2004) propose that the ${ }^{12} \mathrm{C} /{ }^{13} \mathrm{C}$ ratio of hot-core methanol 
should be compared to the corresponding isotopic ratio in $\mathrm{CO}_{2}$ ices, since $\mathrm{CO}$ on cold grains has been shown to react also with atomic oxygen to form solid $\mathrm{CO}_{2}$ (Roser et al. 2001).

Ices are not observed towards "regular" hot cores, but some embedded young stellar objects (YSOs), being at an earlier stage of evolution and harbouring ices in their envelopes, show characteristics implying a small hot core closest to the central object. However, we do not believe it is necessary to observe hot core methanol to get a close connection to the ices. Non-thermal desorption processes like photodesorption (Öberg et al. 2009), exothermic surface reactions (Garrod et al. 2007), or cosmicray-induced heating (Shen et al. 2004) have to be effective in order to explain observed abundances of gas-phase methanol in dark clouds (Willacy \& Millar 1998). These processes should be equally active in the YSO envelopes, resulting in a strong coupling between the gas and the icy grains. The advantages of observing the extended envelope instead of the hot core are that (i) the emission is less likely to be optically thick; (ii) endothermic chemical reactions are unlikely to alter the molecular gas content; (iii) high spatial resolution observations are not required; and (iv) the gas and icy grains coexist and interact.

Observing envelope gas also offers the possibility to compare the ${ }^{12} \mathrm{C} /{ }^{13} \mathrm{C}$ ratio in methanol and $\mathrm{CO}$ gas directly. This has the advantage of not relying on yet another type of grain-surface reaction, here the conversion of $\mathrm{CO}$ to $\mathrm{CO}_{2}$, to leave the ${ }^{12} \mathrm{C} /{ }^{13} \mathrm{C}$ ratio unaltered. The uncertainty arising from analysing optically thick $\mathrm{CO}$ lines can be avoided by observing ${ }^{12} \mathrm{C}^{18} \mathrm{O}$ and ${ }^{13} \mathrm{C}^{18} \mathrm{O}$ instead, since these isotopologues are expected to show a fractionation similar to that in $\mathrm{C}^{16} \mathrm{O}$ (Langer et al. 1984).

In summary, laboratory tests and chemical models indicate that the bulk of interstellar methanol forms from hydrogenation of $\mathrm{CO}$ on ices. Our aim is to test this observationally, primarily by comparing the ${ }^{12} \mathrm{C} /{ }^{13} \mathrm{C}$ ratios in methanol and $\mathrm{C}^{18} \mathrm{O}$ towards several YSOs, which are described in more detail in Sect. 3. The data acquisition and results of the primary analysis are described in Sects. 2 and 4. We verify our method by some excitation and radiative transfer modelling, described in Sect. 5, and finally discuss the results in Sect. 6.

\section{Observations and data reduction}

The observations were performed at $3 \mathrm{~mm}$ wavelength with a SIS mixer receiver on the $20 \mathrm{~m}$ telescope of the Onsala Space Observatory (OSO 20m) during three observing seasons, in the winters of 2006, 2007 and 2009. In each period we observed the ${ }^{12} \mathrm{CH}_{3} \mathrm{OH}$ and ${ }^{13} \mathrm{CH}_{3} \mathrm{OH}$ rotational transition quartets $J=$ $2_{K}-1_{K}$ around 96.7 and $94.4 \mathrm{GHz}$, respectively. In 2009 we also complemented our data set by observing the $J=1-0$ transitions of ${ }^{12} \mathrm{C}^{18} \mathrm{O}$ and ${ }^{13} \mathrm{C}^{18} \mathrm{O}$ in some of the sources. Table 1 presents the transition parameters.

Our source sample consists of nine massive YSOs of different types, or in different stages of evolution, including deeply embedded YSOs, hot cores, and compact $\mathrm{H}_{\text {II }}$ regions, see Sect. 3. The sources were selected based on the availability of either ${ }^{12} \mathrm{CO}_{2}$ and ${ }^{13} \mathrm{CO}_{2}$ ice data or ${ }^{12} \mathrm{C}^{18} \mathrm{O}$ and ${ }^{13} \mathrm{C}^{18} \mathrm{O}$ gas data. Relevant parameters of the observed sources are presented in Table 2.

The observations were performed in the dual beamswitching mode with $\pm 11^{\prime}$ beam separation, through use of a 1600-channel hybrid digital autocorrelation spectrometer at $25 \mathrm{kHz}$ resolution. The $\mathrm{CH}_{3} \mathrm{OH}$ line profiles are well resolved in this setting while the $40 \mathrm{MHz}$ bandwidth easily covers each

\footnotetext{
${ }^{1}$ http://www. astro.uni-koeln.de/cdms/
}

Table 1. Observed transitions.

\begin{tabular}{lccc}
\hline \hline Molecule & Transition & $\begin{array}{c}\text { Frequency } \\
{[\mathrm{GHz}]}\end{array}$ & $\begin{array}{c}E_{\mathrm{up}}^{\mathrm{a}} \\
{[\mathrm{K}]}\end{array}$ \\
\hline${ }^{12} \mathrm{CH}_{3} \mathrm{OH}^{b}$ & $2_{-1} \rightarrow 1_{-1} \mathrm{E}$ & 96.739363 & 4.65 \\
& $2_{0} \rightarrow 1_{0} \mathrm{~A}^{+}$ & 96.741377 & 6.96 \\
& $2_{0} \rightarrow 1_{0} \mathrm{E}$ & 96.744549 & 12.2 \\
& $2_{+1} \rightarrow 1_{+1} \mathrm{E}$ & 96.755507 & 20.1 \\
& $2_{-1} \rightarrow 1_{-1} \mathrm{E}$ & 94.405163 & 4.53 \\
& $2_{0} \rightarrow 1_{0} \mathrm{~A}^{+}$ & 94.407129 & 6.80 \\
${ }^{13} \mathrm{CH}_{3} \mathrm{OH}^{c}$ & $2_{0} \rightarrow 1_{0} \mathrm{E}$ & 94.411016 & 12.0 \\
& $2_{+1} \rightarrow 1_{+1} \mathrm{E}$ & 94.420449 & 20.0 \\
${ }^{12} \mathrm{C}^{18} \mathrm{O}^{c}$ & $1 \rightarrow 0$ & 109.7821734 & 5.27 \\
${ }^{13} \mathrm{C}^{18} \mathrm{O}^{c}$ & $1 \rightarrow 0$ & 104.7114035 & 5.03 \\
\hline
\end{tabular}

Notes. ${ }^{(a)}$ Energies for the E-type $\mathrm{CH}_{3} \mathrm{OH}$ transitions are given relative to the lowest lying E-level, $(J, K)=(1,-1) .{ }^{(b)}$ Data from Mekhtiev et al. (1999). (c) Data from the Cologne Database for Molecular Spectroscopy ${ }^{1}$ (CDMS, Müller et al. 2005).

line quartet. The pointing was typically checked after sunrise and sunset, and was found to be off by less than $7^{\prime \prime}$.

The intensity calibration was done with the chopper wheel method (Ulich \& Haas 1976), generally performed in a cycle of three 60-s signal scans between calibrations. During very stable conditions, each calibration was applied to five signal scans. The focus of our investigation being the isotope ratios, we have taken extra care to check the intensity calibration of our datasets. A new SIS mixer was installed at the OSO $20 \mathrm{~m}$ in early 2008, and although we see no definite trend in our data indicating a performance change, data from the two receivers have not been mixed in the analysis. That is, for each source both ${ }^{12} \mathrm{CH}_{3} \mathrm{OH}$ and ${ }^{13} \mathrm{CH}_{3} \mathrm{OH}$ data used in the analysis are taken with the same receiver, during the same season(s). In addition, the strong ${ }^{12} \mathrm{CH}_{3} \mathrm{OH}$ emission from each source was used to check the relative intensity calibration within and between seasons. The calibration between season 06 and 07 was found to be off by $\sim 30 \%$ for unknown reasons, so data from the later season, where intensities could be confirmed, were used in the analysis. We estimate the systematic calibration errors within each season to be less than $10 \%$, and have consequently added this to the errorbars of the obtained ratios.

The main beam efficiency of OSO $20 \mathrm{~m}$ has been shown to depend not only on frequency, but also on observed elevation, varying between $\sim 0.3$ (115 GHz, low elevation) and $\sim 0.6$ $\left(86 \mathrm{GHz}, 70^{\circ}\right)$. The exact dependency has not been parametrized as a function of frequency and elevation, but since our observations include both circumpolar and low-elevation sources, we have chosen to present our data on the $T_{\mathrm{mb}}$ scale. Integration times are typically $>20 \mathrm{~h}$ for ${ }^{13} \mathrm{CH}_{3} \mathrm{OH}$ and $>1 \mathrm{~h}$ for ${ }^{12} \mathrm{CH}_{3} \mathrm{OH}$ (except for W51 where half the time was sufficient), spanning wide ranges of elevations, so source-specific main beam efficiencies, based on their average elevation during observations, have been used. The used $\eta_{\mathrm{mb}}$ 's are presented in Table 2 . The same $\eta_{\mathrm{mb}}$ has been used for all the methanol lines, since this parameter should differ by less than $3 \%$ between 96.7 and $94.4 \mathrm{GHz}$. The main beam full-width at half-maximum $(F W H M)$ is $39^{\prime \prime}$ at 94.4 GHz and 34" at $109.8 \mathrm{GHz}$.

For three of the observed sources it was not feasible to obtain meaningful methanol isotope ratios due to the low observed $\mathrm{CH}_{3} \mathrm{OH}$ intensity, and for a fourth (NGC 7538 IRS1) we did not reach high enough signal-to-noise ratio in ${ }^{13} \mathrm{CH}_{3} \mathrm{OH}$. However, for two of these (NGC 7538 IRS1 and AFGL 2591) 
Table 2. Observed sources.

\begin{tabular}{lccccccc}
\hline \hline Source & $\begin{array}{c}\text { RA (J2000.0 }) \\
(\mathrm{h} \mathrm{m} \mathrm{s})\end{array}$ & $\begin{array}{c}\text { Dec }(\mathrm{J} 2000.0) \\
\left({ }^{\circ}, "\right)\end{array}$ & $\begin{array}{c}v_{\mathrm{LSR}} \\
\left(\mathrm{km} \mathrm{s}^{-1}\right)\end{array}$ & $\begin{array}{c}\text { Distance } \\
(\mathrm{kpc})\end{array}$ & $\begin{array}{c}\eta_{\mathrm{mb}} \\
\left(\mathrm{CH}_{3} \mathrm{OH}\right)\end{array}$ & $\begin{array}{c}\eta_{\mathrm{mb}} \\
\left({ }^{12} \mathrm{C}^{18} \mathrm{O}\right)\end{array}$ & $\begin{array}{c}\eta_{\mathrm{mb}} \\
\left({ }^{13} \mathrm{C}^{18} \mathrm{O}\right)\end{array}$ \\
\hline W51 e1/e2 & 192344.0 & +143029 & +57 & $7^{a}$ & 0.43 & - & - \\
NGC 2264 IRS1 & 064110.1 & +092936 & +8 & $0.9^{b}$ & 0.42 & - & - \\
DR21 (OH) & 203900.9 & +422238 & -3 & $3^{c}$ & 0.51 & - & - \\
NGC 7538 IRS9 & 231401.7 & +612720 & -57 & $2.7^{d}$ & 0.52 & 0.46 & 0.48 \\
S140 IRS1 & 221918.2 & +631847 & -7 & $0.9^{e}$ & 0.52 & 0.46 & 0.48 \\
NGC 7538 IRS1 & 231345.4 & +612811 & -57 & $2.7^{d}$ & 0.40 & - & - \\
AFGL 2591 & 202924.7 & +401119 & -6 & $1^{f}$ & 0.47 & - & - \\
\hline NGC 2024 IRS2 & 054145.8 & -015434 & +12 & & & & \\
W3 IRS5 & 022540.6 & +620551 & -39 & & & & \\
\hline
\end{tabular}

Notes. The sources in the second part of the table, below the line, did not have strong enough methanol emission to be included in the analysis, and therefore the parameters relevant for the analysis have been omitted.

References. ${ }^{(a)}$ Genzel et al. (1981); ${ }^{(b)}$ Neri et al. (1993); ${ }^{(c)}$ Very uncertain, see Pipenbrink \& Wendker (1988); ${ }^{(d)}$ Moscadelli et al. (2009); ${ }^{(e)}$ Crampton \& Fisher (1974); ${ }^{(f)}$ Very uncertain, see van der Tak et al. (1999) .

the ${ }^{12} \mathrm{CH}_{3} \mathrm{OH}$ signals have high enough signal-to-noise ratio to be included in part of the analysis, see Sect. 4.2.

Towards two of the sources, NGC 2024 IRS2 and NGC 2264 IRS1, attempts were also made to observe the $J=7_{K}-6_{K}$ transitions of ${ }^{12} \mathrm{CH}_{3} \mathrm{OH}$ and ${ }^{13} \mathrm{CH}_{3} \mathrm{OH}$, around 338 and $330 \mathrm{GHz}$ respectively, with the APEX (Atacama Pathfinder EXperiment) telescope ${ }^{2}$. However, no ${ }^{13} \mathrm{CH}_{3} \mathrm{OH}$ signals were detected above the recorded noise of $\sim 25 \mathrm{mK}$ RMS.

Linear baselines were subtracted from individual scans before averaging them, using baseline RMS values as weights. In addition, the ${ }^{13} \mathrm{CH}_{3} \mathrm{OH}$ spectra have been smoothed by a five channel wide triangular weighting function prior to analysis and presentation. All data reduction was performed using the spectral line data reduction software $\mathrm{XS}^{3}$, developed by Per Bergman at OSO.

\section{The sources}

Four of our observed sources, namely NGC 2264 IRS1; NGC 7538 IRS 9; S140 IRS1; and AFGL 2591, are massive YSOs at an early stage of evolution with at most a very modest hot core, not yet surrounded by $\mathrm{H}_{\text {II }}$ regions, but embedded in dust that is cold enough to harbour a variety of ices, as shown by ISO and Spitzer observations (Gibb et al. 2004; Pontoppidan et al. 2008). The envelope of the massive YSO at NGC 7538 IRS1 also contains ices according to these observations, but its core is bipolar and free-free emission indicates the presence of an ultracompact $\mathrm{H}_{\text {II }}$ region, or possibly an ionized jet (Sandell et al. 2009). Similarly, the infrared sources e1 and e2 are associated with the W51d ultracompact $\mathrm{H}$ II region. They are however buried in the molecular cloud W51, which exhibits emission from a large variety of complex organic molecules, typical of hot cores (Remijan et al. 2004). Infrared spectroscopy of sources e1 and 2 by Barbosa et al. (2008) shows a mixture of evolutionary stages, including an $\mathrm{O} 3$ star responsible for the ionised gas and a massive YSO that is still surrounded by an accretion disk. No ices have been observed towards this part of W51.

DR21 $(\mathrm{OH})$ is not easily classified. Its position, about $3^{\prime}$ north of DR21, is primarily associated with $\mathrm{OH}, \mathrm{H}_{2} \mathrm{O}$ and

\footnotetext{
2 http://www . apex-telescope.org/

3 http://www . chalmers.se/rss/oso-en/observations/

data-reduction-software
}

methanol masers, and it hosts several massive YSOs, very similar in evolutionary state to the three sources described above (Chandler et al. 1993). However, no ices have been reported along the line-of-sight towards it. A possible explanation is that the dust envelopes have been externally heated by the nearby, and more evolved, massive star-forming region of DR2 1 itself (cf. Araya et al. 2009).

It should be noted that the typical scale for the YSOs in question is of the order of one arcsecond or less, while the hot cores have diameters of up to $\sim 5^{\prime \prime}$. In contrast, their gas and dust envelopes are extended, typically filling the OSO $20 \mathrm{~m}$ beam. Several of the sources were observed in higher excitation methanol and formaldehyde by van der Tak et al. (2000), and two of them were reported to show evidence for significantly increased methanol abundance towards the centre, AFGL 2591 and NGC 7538 IRS1, presumably due to thermal evaporation of ice mantels. Thus, while the bulk of the here observed lowexcitation methanol is unlikely to originate from thermally evaporated ices near the central object when the abundance is constant throughout the source, a significant contribution cannot be excluded in the case of the above mentioned sources and W51 e1/e2. This should however not effect the ${ }^{12} \mathrm{C} /{ }^{13} \mathrm{C}$ ratio in methanol, as long as it is formed by the same mechanism. Moreover, in some cases the beam encompasses additional compact sub-sources/YSOs of the same type.

\section{Results and data analysis}

Figures 1-5 show the observed methanol spectra towards the sources where ${ }^{13} \mathrm{CH}_{3} \mathrm{OH}$ was observed. Note that the line quartets in $\mathrm{CH}_{3} \mathrm{OH}$ and ${ }^{13} \mathrm{CH}_{3} \mathrm{OH}$ are not expected to be perfectly aligned since the relative frequencies are not identical (see Table 1). This is illustrated in the figures by the vertical lines, marking the expected positions of the lines in the adopted velocity frame.

As expected, the peak intensities of the individual lines of both isotopes decrease with increasing energy, the highest energy ${ }^{13} \mathrm{CH}_{3} \mathrm{OH}$ line being completely buried in noise in all sources except W51 e1/e2. Because of this incompleteness in the dataset, and for consistency, this line has been excluded from the analysis. Gaussian line parameters for observed methanol lines are presented in Table A.1. The line-widths are typically $5 \mathrm{~km} \mathrm{~s}^{-1}$ or less, except towards W51 e1/e2, where the three 


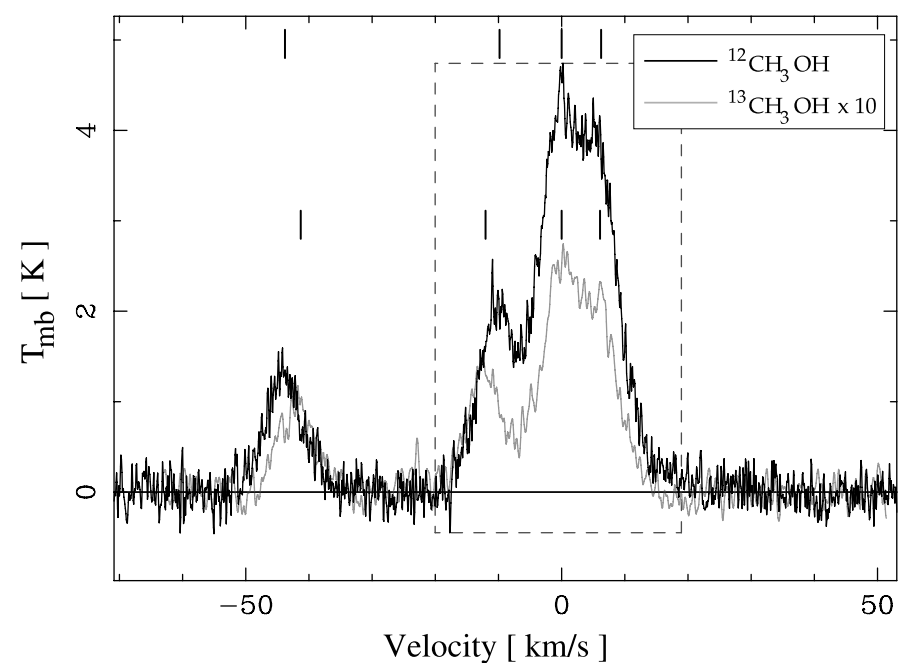

Fig. 1. The ${ }^{12} \mathrm{CH}_{3} \mathrm{OH}$ and ${ }^{13} \mathrm{CH}_{3} \mathrm{OH}$ spectra towards W51 e1/e2. For both isotopologues the velocity has been set to zero at the frequency of the lowest energy line (see Table 1), and the upper and lower sets of vertical lines mark the expected line velocities for ${ }^{12} \mathrm{CH}_{3} \mathrm{OH}$ and ${ }^{13} \mathrm{CH}_{3} \mathrm{OH}$, respectively. The dashed box marks the velocity interval over which the intensities, presented in Table 3, have been integrated. Note that the intensity of the ${ }^{13} \mathrm{CH}_{3} \mathrm{OH}$ spectrum has been scaled up by a factor of 10 .

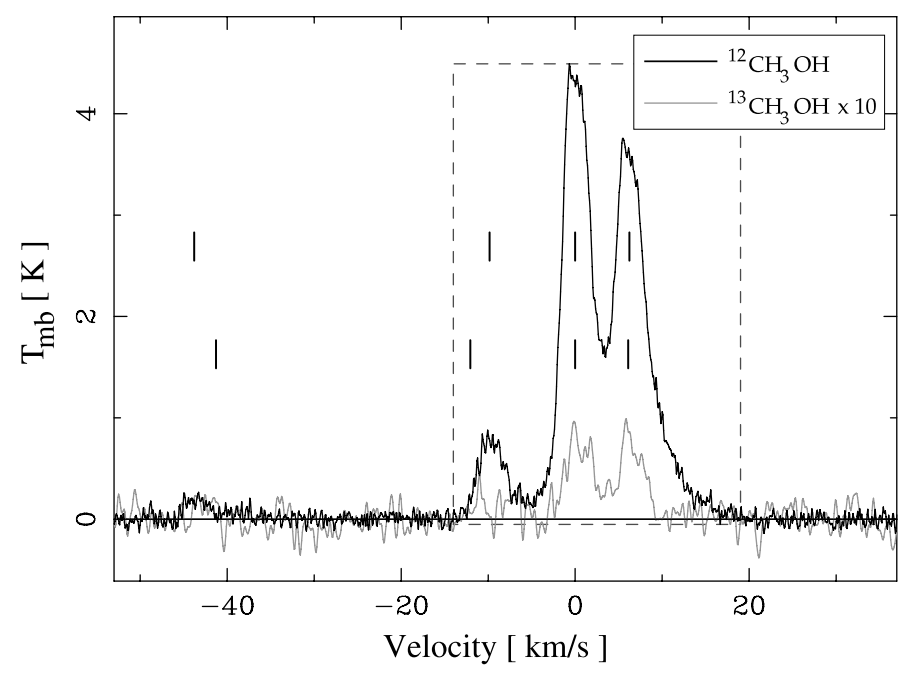

Fig. 2. The ${ }^{12} \mathrm{CH}_{3} \mathrm{OH}$ and ${ }^{13} \mathrm{CH}_{3} \mathrm{OH}$ spectra towards NGC 2264 IRS1. Graph key is the same as in Fig. 1.

main lines are wide enough to give substantial overlap, and Gaussian fits yield line-widths of 6 to $8 \mathrm{~km} \mathrm{~s}^{-1}$. The observed differences in both line intensities and widths between W51 $\mathrm{e} 1 / \mathrm{e} 2$ and the other sources are readily explained by evolutionary differences, the sub-sources e $1 / \mathrm{e} 2$ being known hot cores, see Sect. 3, and W51 being the most distant and least well resolved of our targets.

\section{1. ${ }^{12} \mathrm{C} /{ }^{13} \mathrm{C}$ isotope ratio}

We propose that the observed integrated intensity ratio $I\left({ }^{12} \mathrm{CH}_{3} \mathrm{OH}\right) / I\left({ }^{13} \mathrm{CH}_{3} \mathrm{OH}\right)$ reflects the isotope ratio of the gas to within $5 \%$, assuming that (i) the level population distributions of ${ }^{12} \mathrm{CH}_{3} \mathrm{OH}$ and ${ }^{13} \mathrm{CH}_{3} \mathrm{OH}$ are described by Boltzmann functions at a common rotation temperature; (ii) their spatial distributions coincide; and (iii) the emission is optically thin.

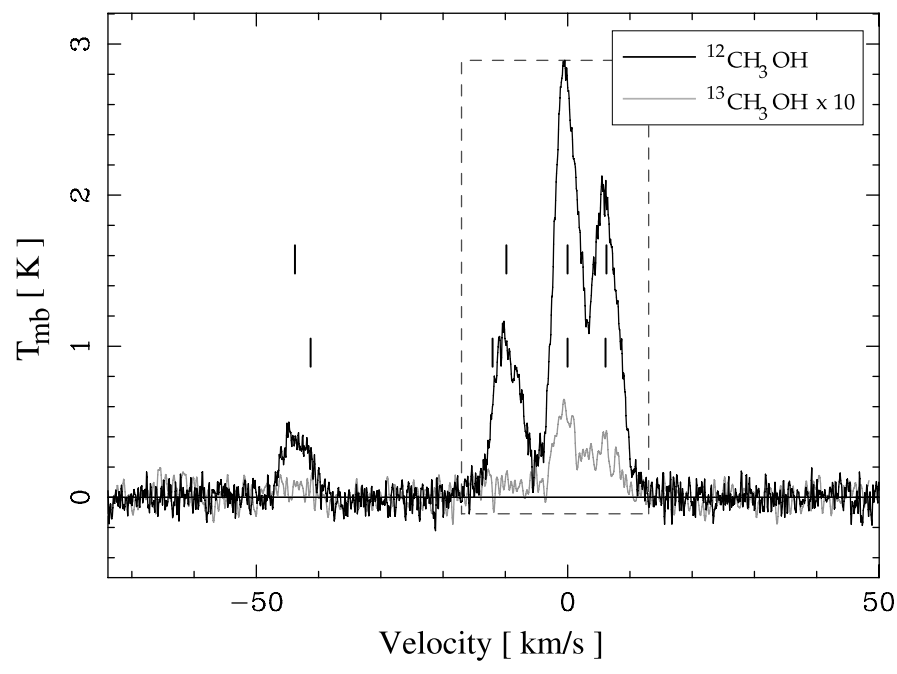

Fig. 3. The ${ }^{12} \mathrm{CH}_{3} \mathrm{OH}$ and ${ }^{13} \mathrm{CH}_{3} \mathrm{OH}$ spectra towards DR $21(\mathrm{OH})$. Graph key is the same as in Fig. 1.

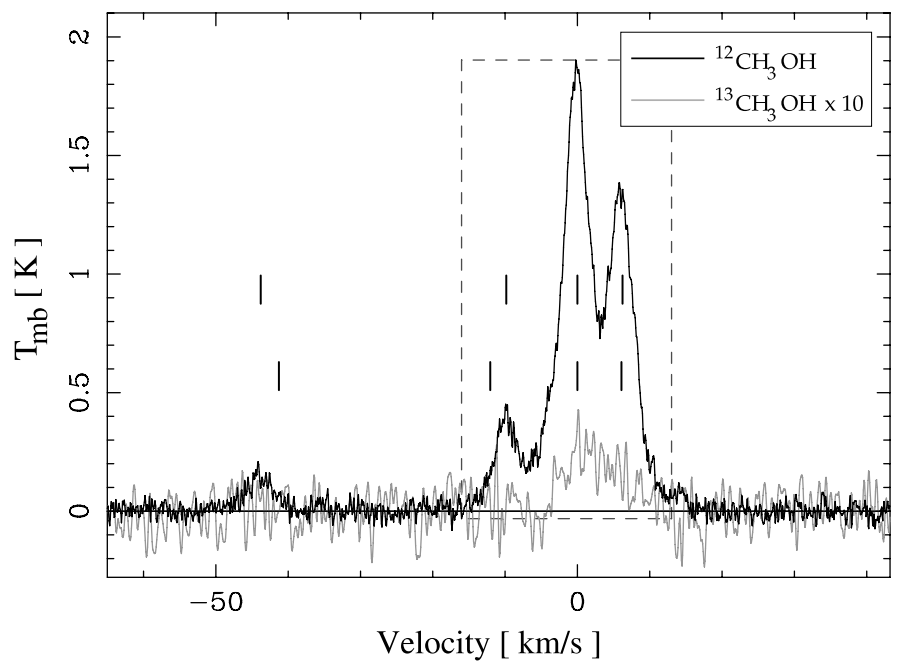

Fig. 4. The ${ }^{12} \mathrm{CH}_{3} \mathrm{OH}$ and ${ }^{13} \mathrm{CH}_{3} \mathrm{OH}$ spectra towards NGC 7538 IRS9. Graph key is the same as in Fig. 1.

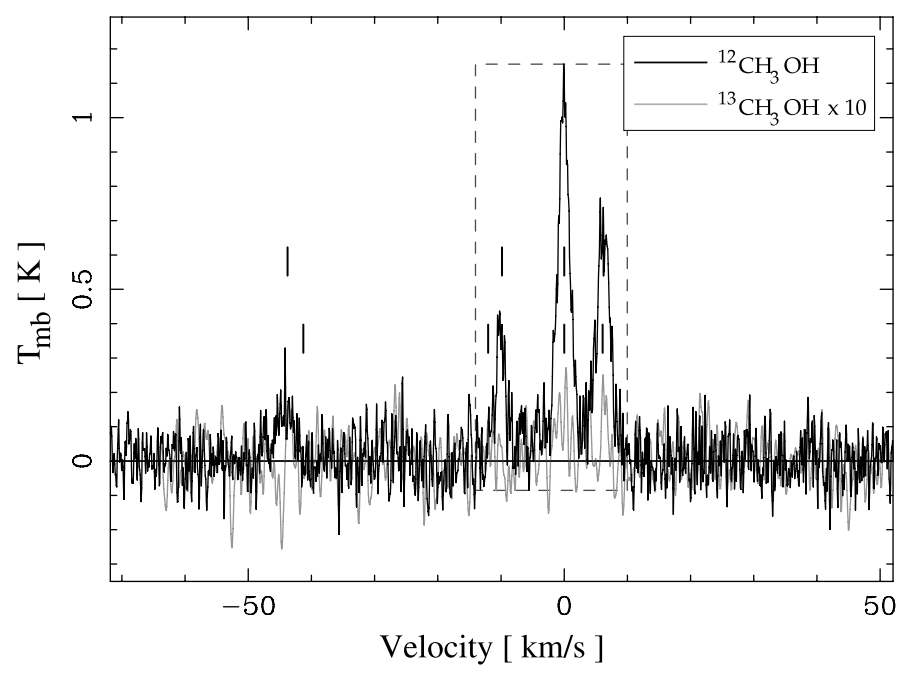

Fig. 5. The ${ }^{12} \mathrm{CH}_{3} \mathrm{OH}$ and ${ }^{13} \mathrm{CH}_{3} \mathrm{OH}$ spectra towards $\mathrm{S} 140$ IRS1. Graph key is the same as in Fig. 1. 
Table 3. Observed ${ }^{12} \mathrm{C} /{ }^{13} \mathrm{C}$ integrated intensity ratios.

\begin{tabular}{lccccccc}
\hline \hline Source & $\begin{array}{c}I\left({ }^{12} \mathrm{CH}_{3} \mathrm{OH}\right) \\
\left(\mathrm{K} \mathrm{km} \mathrm{s}^{-1}\right)\end{array}$ & $\begin{array}{c}I\left({ }^{13} \mathrm{CH}_{3} \mathrm{OH}\right) \\
\left(\mathrm{mK} \mathrm{km} \mathrm{s}^{-1}\right)\end{array}$ & $R\left(\mathrm{CH}_{3} \mathrm{OH}\right)$ & $\begin{array}{c}I\left({ }^{12} \mathrm{C}^{18} \mathrm{O}\right) \\
\left(\mathrm{K} \mathrm{km} \mathrm{s}^{-1}\right)\end{array}$ & $\begin{array}{c}I\left({ }^{13} \mathrm{C}^{18} \mathrm{O}\right) \\
\left(\mathrm{mK} \mathrm{km} \mathrm{s}^{-1}\right)\end{array}$ & $R\left(\mathrm{C}^{18} \mathrm{O}\right)$ & $R\left(\mathrm{CO}_{2, \text { ice }}\right)$ \\
\hline W51 e1/e2 & $73.7 \pm 0.2$ & $4120 \pm 30$ & $18 \pm 3$ & $18.8 \pm 0.10^{a}$ & $413 \pm 14^{a}$ & $46 \pm 2^{a}$ & - \\
NGC 2264 IRS1 & $40.9 \pm 0.2$ & $722.9 \pm 60$ & $57 \pm 9$ & $5.11 \pm 0.02^{a}$ & $90.5 \pm 5.4^{a}$ & $56 \pm 3^{a}$ & $131 \pm 21^{b}$ \\
DR21 (OH) & $29.2 \pm 0.1$ & $534 \pm 15$ & $55 \pm 8$ & $7.09 \pm 0.02^{a}$ & $123 \pm 7^{a}$ & $58 \pm 3^{a}$ & - \\
NGC 7538 IRS9 & $17.5 \pm 0.1$ & $250 \pm 38$ & $70 \pm 15$ & $4.18 \pm 0.05$ & $61.9 \pm 9.6$ & $68 \pm 14$ & $80 \pm 11^{c}$ \\
S140 IRS1 & $5.83 \pm 0.09$ & $72 \pm 15$ & $81 \pm 20$ & $7.67 \pm 0.05$ & $92.6 \pm 8.2$ & $83 \pm 14$ & $111 \pm 11^{b}$ \\
\hline
\end{tabular}

Notes. Integrated intensities are calculated from the main-beam corrected spectra and have $1 \sigma$ errors attached, while the ratio errors include an additional $10 \%$ calibration error.

References. ${ }^{(a)}$ Langer \& Penzias (1990); ${ }^{(b)}$ Gibb et al. (2004); ${ }^{(c)}$ Boogert et al. (2000);

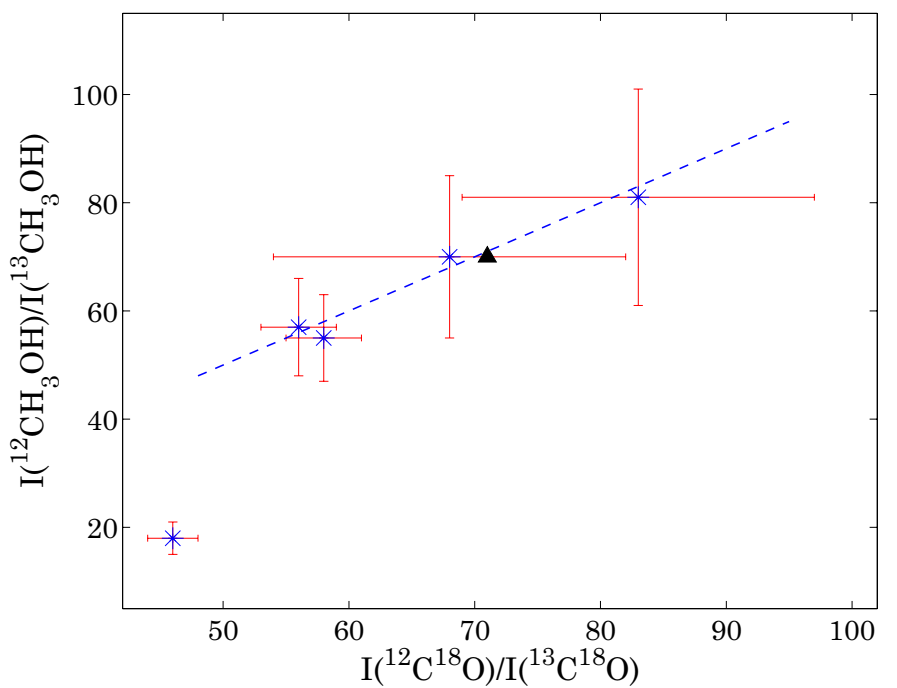

Fig. 6. The relation between the ${ }^{12} \mathrm{C} /{ }^{13} \mathrm{C}$ isotopic ratio in $\mathrm{C}^{18} \mathrm{O}$ and $\mathrm{CH}_{3} \mathrm{OH}$ for the observed sources (stars), with the dashed line representing a 1:1 relation plotted to facilitate interpretation. Ratios and errors are those found in Table 3, the source with the relatively low methanol ratio is $\mathrm{W} 51 \mathrm{e} 1 / \mathrm{e} 2$. The black triangle marks the ${ }^{12} \mathrm{C} /{ }^{13} \mathrm{C}$ ratio in methanol versus solid $\mathrm{C}^{16} \mathrm{O}$ towards NGC 7538 IRS9, see Sect. 6.

These intensities, integrated over the velocity intervals marked in Figs. 1-5, covering the line triplets with lowest upper state energies, are presented in Table 3 together with the resulting ratios. This table also presents the corresponding parameters from the $\mathrm{C}^{18} \mathrm{O}$ observations, with integration intervals common to ${ }^{12} \mathrm{C}^{18} \mathrm{O}$ and ${ }^{13} \mathrm{C}^{18} \mathrm{O}$. For three of the sources $\mathrm{C}^{18} \mathrm{O}$ ratios have been calculated from integrated intensities presented by Langer \& Penzias (1990). For comparison, the ${ }^{12} \mathrm{C} /{ }^{13} \mathrm{C}$ isotopic ratio in solid $\mathrm{CO}_{2}$ has also been included: see further discussion in Sect. 6.

Figure 6 illustrates the relationship between the observed ${ }^{12} \mathrm{C} /{ }^{13} \mathrm{C}$ ratios in $\mathrm{CH}_{3} \mathrm{OH}$ and $\mathrm{C}^{18} \mathrm{O}$, compared to a $1: 1$ correlation. Four of the sources do display a striking similarity in their ${ }^{12} \mathrm{C} /{ }^{13} \mathrm{C}$ ratios between methanol and carbon monoxide, while one source, W51 e1/e2, has a comparatively low integrated intensity ratio in methanol. This can be anticipated already from a quick eye-inspection of the spectra in Fig. 1, the signal of the rarer isotope being so strong compared to the common one. If the isotope abundance ratio were similar to the intensity ratios observed in methanol in the other sources, then the relatively strong ${ }^{13} \mathrm{CH}_{3} \mathrm{OH}$ emission would imply that the optical depth in the strongest ${ }^{12} \mathrm{CH}_{3} \mathrm{OH}$ lines is probably greater than unity. On the other hand the similarity in profile shapes for the two isotopologues in W51 e1/e2 suggests that the optical depth is not large. Thus it is not clear whether one of the basic assumptions in our analysis is violated or not in the case of this source.

\section{2. $E$ to $A$ methanol ratio}

There are two types of methanol, owing to the possible combinations of nuclear spin alignment in the three H-atoms of the methyl-group. When these spins are parallel we have A-methanol and when they are not, the total nuclear spin adding up to $1 / 2$, we have E-methanol. Since nuclear spins interact so weakly with rotation, electrical force etcetera, there are no allowed radiative or collisional transitions between E- and Atype methanol. Hence, in the context of excitation the E and A methanol are often treated as two separate species, expected to exist at a close to 1:1 ratio based on the nuclear-spin weights and the two degenerate forms of E-methanol.

The temperature that describes the relative population of non-interacting spin-types of a molecule is called the nuclear spin temperature, $T_{\text {spin }}$. For methanol, the equilibrium E/A ratio is related to the spin temperature via weighted sums over all Eand A-levels, according to

$E / A=\frac{\sum_{i \in E} g_{i} \exp \left(-\frac{E_{i}}{k T_{\text {spin }}}\right)}{\sum_{j \in A} g_{j} \exp \left(-\frac{E_{j}}{k T_{\text {spin }}}\right)}$

where $g_{i / j}$ is the statistical weight $(2 J+1)$ and $E_{i / j}$ is the energy of the level.

It is expected that the formation process, and the environment it takes place in, control the initial level population distribution within a molecular species. For methanol, the ground state of E-methanol, $(J, K)=(1,-1)$, lies $7.9 \mathrm{~K}$ above the ground state of A-methanol, $(J, K)=(0,0)$, so if the formation results in a population distribution thermalized at a low temperature, an overabundance of A-methanol is created. This might be expected to be the case for methanol formation by successive hydrogenation of $\mathrm{CO}$, which has been shown to be most effective at physical temperatures of about $10-15 \mathrm{~K}$ on $\mathrm{CO}: \mathrm{H}_{2} \mathrm{O}$ ice mixtures or pure solid CO (Watanabe et al. 2004). Such an overabundance of A-methanol is then preserved through changing excitation conditions, and measurable through the nuclear spin temperature. Equation (2) shows that the E/A ratio decreases by more than $0.5 \%$ from its high-temperature equilibrium value of one for $T_{\text {spin }} \lesssim 37 \mathrm{~K}$, and is most sensitive to spin temperatures around 10-20 K (see also Fig. 7). In cometary research it is common 
Table 4. Temperatures and E/A ratios

\begin{tabular}{lccc}
\hline \hline Source & $T_{\text {rot }}^{\mathrm{a}}[\mathrm{K}]$ & $T_{\text {spin }}[\mathrm{K}]$ & $\mathrm{E} / \mathrm{A}$ \\
\hline W51 e1/e2 & $29^{b}$ & $16^{b}$ & $0.88 \pm 0.05$ \\
NGC 2264 IRS1 & 5 & $>37^{c}$ & $1.24 \pm 0.19$ \\
DR21 (OH) & 10 & $>37^{c}$ & $1.29 \pm 0.10$ \\
NGC 7538 IRS9 & 7 & $>22^{d}$ & $1.08 \pm 0.12$ \\
S140 IRS1 & 12 & $>23^{d}$ & $1.03 \pm 0.07$ \\
NGC 7538 IRS1 & 8 & $>36^{d}$ & $1.09 \pm 0.10$ \\
AFGL 2591 & 9 & 10 & $0.71 \pm 0.06$ \\
\hline
\end{tabular}

Notes. ${ }^{(a)}$ From methanol E-lines. ${ }^{(b)}$ Using ${ }^{13} \mathrm{CH}_{3} \mathrm{OH} .{ }^{(c)}$ Lower limit set at $\mathrm{E} / \mathrm{A}=0.995 .{ }^{(d)}$ Lower limit corresponding to lower limit $\mathrm{E} / \mathrm{A}$ value.

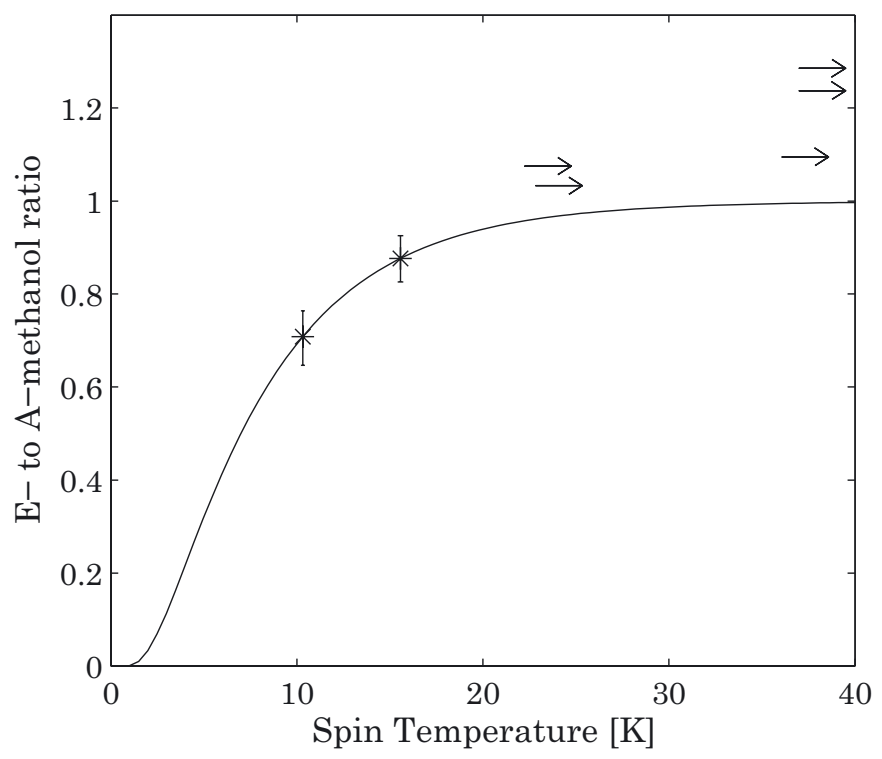

Fig. 7. The equilibrium E- to A- methanol ratio as a function of nuclear spin temperature (solid curve). Ratios $<1$ observed towards our sources are plotted as stars according to this relation, errorbars indicating effect of a $10 \%$ difference in the assumed $T_{\text {ex }}$ for A-methanol. Horizontal arrows represent sources where ratios $>1$ according to the $y$-scale, and are drawn from the equivalent spin temperature of the lower errorbar.

for the spin temperatures of molecules like water, ammonia, and methane to be interpreted as the physical temperature at which the molecules formed or condensed (e.g. Kawakita et al. 2006; Kawakita \& Kobayashi 2009), although this is a subject under debate (Crovisier 2007).

The relative population of E- and A-type methanol can be altered by other processes following the formation, equilibrating the E/A ratio at another characteristic temperature. In the molecular interstellar medium, the most likely such process is proton exchange reactions with $\mathrm{H}_{3}^{+}$and $\mathrm{HCO}^{+}$. However, the expected lifetime of methanol molecules in the observed type of sources $\left(\sim 10^{5}\right.$ years $)$ is likely to be shorter than the timescale for these proton exchange reactions, see Appendix A. An overabundance of A-methanol created at formation should therefore be preserved.

If the nuclear spin temperature reflects the physical formation temperature, then measurement of the E/A ratio provides another observational test of the hypothesis that interstellar methanol is forming from $\mathrm{CO}$ on cold grain surfaces.

Using the same procedure as Menten et al. (1988), we derive a $T_{\text {rot }}$ for each source from rotation diagrams of the ${ }^{12} \mathrm{CH}_{3} \mathrm{OH}$
E-lines $\left({ }^{13} \mathrm{CH}_{3} \mathrm{OH}\right.$ for W51 e1/e2), and assume that the population distributions of both $\mathrm{E}$ and $\mathrm{A}$ methanol are described by this temperature. Figures A.1 and A.2 show the rotational diagrams and the temperatures are summarized in Table 4. The resulting E/A column density ratios are below one for two sources, W51 e1/e2 and AFGL 2591. In Fig. 7 the equilibrium E/A ratio is plotted as a function of nuclear spin temperature (Eq. (2)), together with these measured ratios. Observational errors due to noise are small in the E/A ratio, and calibration uncertainties do not affect the ratio since both $\mathrm{E}$ and $\mathrm{A}$ data are from the same spectrum. Instead we have estimated the effect of a difference in excitation between E- and A-methanol. Thus, the error bars in the figure show the effect of a $10 \%$ higher or lower $T_{\text {ex }}$ for Amethanol as compared to the $T_{\text {rot }}$ derived from the E-methanol data. For a discussion evaluating the rotation diagram method as compared to non-LTE modelling, see Sect. 5.

Figure 7 demonstrates that two of the observed sources have measured $\mathrm{E} / \mathrm{A}$ ratios consistent with nuclear spin temperatures below $20 \mathrm{~K}, T_{\text {spin }}=10$ and $16 \mathrm{~K}$. This is right around the temperature range where the methanol production from $\mathrm{CO}$ hydrogenation on grains is most effective (Watanabe et al. 2004), and thus consistent with this formation theory. However, the other five sources display E/A ratios consistent with population equilibration at a higher temperature. Low E/A ratios have indeed been observed before, with values of $0.69-0.87$ in dark clouds (Friberg et al. 1988) and 0.50-0.77 in cool clumps of Orion (Menten et al. 1988), but not much further analysis has been presented. The spin temperatures of methanol have also been investigated towards comet Hale-Bopp, where the obtained lower limits are at the high end of, or above, the temperature range within which the methanol formation from $\mathrm{CO}$ hydrogenation on grains has been shown to be effective (Pardanaud et al. 2007).

\section{RADEX modelling}

In order to validate the results presented in Section 4, we want to investigate the excitation and radiative transfer of methanol in the kinds of environments observed. For this we use the RADEX code (van der Tak et al. 2007) to model the level populations and line intensities of methanol molecules. The molecular datafile includes all transitions involving torsional-rotational transitions for $J \leq 12$ and $v_{t}=0,1,2$ from Mekhtiev et al. (1999), 72145 transitions in total, involving 2742 levels. Collision rates for para- $\mathrm{H}_{2}$ collisions with methanol have been adapted from Pottage et al. (2004) for relatively low-lying rotational states in $v_{t}=0$. Additional collision rates have been guessed by scaling the line strengths of all other radiatively allowed transitions to a generic downward rate coefficient of $1.0 \times 10^{-11} \mathrm{~cm}^{3} \mathrm{~s}^{-1}$.

The non-LTE excitation calculations are used to investigate the crucial assumption of equal excitation temperatures in $3 \mathrm{~mm}$ transitions of $\mathrm{CH}_{3} \mathrm{OH}$ and ${ }^{13} \mathrm{CH}_{3} \mathrm{OH}$. For illustration, we consider models of the W 51 complex with kinetic temperature $T_{\text {kin }} \sim 100 \mathrm{~K}$ and densities $n\left(\mathrm{H}_{2}\right) \gtrsim 10^{5} \mathrm{~cm}^{-3}$. These models include a continuum radiation field derived directly from the observed spectral energy distribution at infrared and sub-mm wavelengths. These calculations reproduce well the observed relative intensities, but they display a wide range of excitation temperatures for individual transitions. In one example at $T_{\text {kin }}=100 \mathrm{~K}$, the excitation temperatures of the strongest lines between 92 and $98 \mathrm{GHz}$ have values from -270 to $+640 \mathrm{~K}$, but the average rotation temperature of the 49 most populous levels in the model is well defined and subthermal at $33 \mathrm{~K}$. The wide spread in excitation temperatures has been noted before, e.g. in dark clouds by Friberg et al. (1988) and in a comparison of LTE and 
LVG methanol modelling by Bachiller et al. (1998), and is a physical effect, not a numerical artefact of the model. It reflects a shifting balance between collisions at the kinetic temperature $T_{\text {kin }}$ and radiative excitation. Indeed it is very difficult to interpret the excitation temperatures because so many levels are coupled to each other by collisional and radiative processes operating at quite different temperatures. Note that the suprathermal excitation of some transitions foremost is a result of the complex energy level structure, but that the effect may be significantly enhanced by the pumping of an external radiation field.

The spread in excitation temperatures generally decreases with model kinetic temperature, but remains significant. The other sources are typically well fit by RADEX models at kinetic temperatures and $\mathrm{H}_{2}$ densities of $15-30 \mathrm{~K}$ and $10^{5}-10^{6} \mathrm{~cm}^{-3}$, and methanol column densities ranging from $2 \times 10^{14}$ to $2 \times$ $10^{15} \mathrm{~cm}^{-2}$. Modelled optical depths are as high as 0.3 for the highest intensity line, but since the integrated intensity ratio is computed over the group of three lines, this would infer an offset of $<15 \%$ between the methanol isotopologue ratio and the integrated intensity ratio illustrated in Fig. 6.

In summary, the assumption of a methanol level population distribution described by a single temperature is not valid for our sources. However, the assumption does not need to be that general for the observed integrated intensity ratio to be a good measure of the isotope ratio. It is sufficient that the population distribution between the two levels involved in each observed transition should be described by an excitation temperature that is common to both isotopologues, ${ }^{12} \mathrm{CH}_{3} \mathrm{OH}$ and ${ }^{13} \mathrm{CH}_{3} \mathrm{OH}$. The RADEX models suggest that this is the case: the excitation temperatures for each transition differ by less than 5\% between modelled ${ }^{12} \mathrm{CH}_{3} \mathrm{OH}$ and ${ }^{13} \mathrm{CH}_{3} \mathrm{OH}$ in the modelled range of conditions for the low temperature envelopes (see above). Even though the rotation diagram method gives a false impression of a single temperature describing the population distribution, the column density derived using this temperature is not necessarily far from the truth, depending on the number and distribution of transitions used in the fit. In other words, while a derived rotation temperature has no physical meaning and is rather useless for predicting the population of a specific level, in some cases it does describe an appropriate "average" population distribution that corresponds to the same total number of molecules as the more detailed one it represents.

\section{Discussion}

In employing isotopic ratios as a tracer of the formation path of methanol, the most useful comparison would be between solid-state $\mathrm{CO}$, methanol, and preferably also formaldehyde, $\mathrm{H}_{2} \mathrm{CO}$. Unfortunately, the telescopes/instruments available up to now are not sensitive to the rarer isotopes of the hydrated ices. However, ${ }^{13} \mathrm{C}^{16} \mathrm{O}$ has been observed in its solid form towards one of our sources, NGC 7538 IRS 9, at an isotopic ${ }^{12} \mathrm{CO} /{ }^{13} \mathrm{CO}$ ratio of $71 \pm 15$ (Boogert et al. 2002). This measurement has been added to Fig. 6 in the form of a black triangle at the gas-phase methanol ratio towards the same source, showing how similar the ${ }^{12} \mathrm{C} /{ }^{13} \mathrm{C}$ isotopic ratios are in gas-phase $\mathrm{C}^{18} \mathrm{O}$, solid $\mathrm{CO}$, and gas-phase methanol.

To justify this test being conducted on gas-phase methanol, it is desirable to establish whether the ${ }^{12} \mathrm{C} /{ }^{13} \mathrm{C}$ ratio is likely to have been altered since formation, either by selective desorption or by gas-phase reactions. There is very little detailed information available about the effects of isotope fractionation at various types of desorption processes, except in the H/D case (e.g. Willacy \& Millar 1998; Amiaud et al. 2007). The ${ }^{13} \mathrm{C}$ fractionation in $\mathrm{CO}$ from thermal desorption has been modelled at temperatures $\leq 12 \mathrm{~K}$ where ${ }^{12} \mathrm{CO}$ was found to desorb $\sim 8 \%$ more efficiently than ${ }^{13} \mathrm{CO}$ (Young \& Schauble 2011), while a laboratory study of thermal $\mathrm{CO}$ desorption from $\mathrm{CO} / \mathrm{O}_{2}$ ice arrives at only a few percent difference (Acharyya et al. 2007). Assuming that the desorption fractionation for methanol is governed by the difference in molecular mass, which seems to be likely based on the mentioned studies, the effect should not be more than a few percent.

Because of the four bonds to the $\mathrm{C}$ atom in methanol, gasphase ${ }^{12} \mathrm{C} /{ }^{13} \mathrm{C}$ substitution is not expected to be efficient. The molecule has to be broken apart, which is most likely to happen from collisions with $\mathrm{H}_{3}^{+}$or $\mathrm{HCO}^{+}$as discussed in Appendix A, and then re-formed from another carbon. The timescale for this process cannot be shorter than for forming methanol from scratch in the gas-phase. Therefore we expect the ${ }^{13} \mathrm{C} /{ }^{12} \mathrm{C}$ ratio in methanol to be controlled by the formation process, whether this is on grains or in the gas-phase.

Following the suggestion of Charnley et al. (2004), we have also made comparisons of the gas-phase methanol to the ${ }^{12} \mathrm{C} /{ }^{13} \mathrm{C}$ ratios in $\mathrm{CO}_{2}$ ices, here as observed by ISO (Boogert et al. 2000; Gibb et al. 2004). These ratios are given in the rightmost column of Table 3. However, they tend to be substantially higher than the corresponding ratios in both gas-phase methanol and $\mathrm{C}^{18} \mathrm{O}$, which raises some questions regarding the formation of $\mathrm{CO}_{2}$ on interstellar grains. If the main production in these sources is by almost barrierless reactions of adsorbed $\mathrm{O}$-atoms with $\mathrm{CO}$, argued for by Roser et al. (2001), the ${ }^{12} \mathrm{C} /{ }^{13} \mathrm{C}$ isotopic ratios should be low, reflecting the fractionation in cold $\mathrm{CO}$ gas. However, in the envelopes of the YSOs we have observed, the ices very likely have been energetically processed by e.g. ion irradiation, which also has been shown to produce solid $\mathrm{CO}_{2}$. In this case, several different types/mixtures of $\mathrm{C}$ - and $\mathrm{O}$-containing ices may contribute to the $\mathrm{CO}_{2}$ production (Ioppolo et al. 2009), not necessarily resulting in an isotope ratio similar to that in $\mathrm{CO}$.

In our sources, $\mathrm{CO}$ is typically the major carbon bearing species. The effect of ${ }^{13} \mathrm{C}$ fractionation on the ${ }^{12} \mathrm{CO} /{ }^{13} \mathrm{CO}$ ratio decreases with the fraction of elemental carbon locked up in $\mathrm{CO}$, but fractionation would still have a substantial impact on other species formed in the gas-phase, the so called "carbon isotope pool", where ${ }^{13} \mathrm{C}$ would be depleted. Since there is no effective gas-phase mechanism to fractionate ${ }^{13} \mathrm{C}$ in methanol or in its ionic precursors, its ${ }^{12} \mathrm{C} /{ }^{13} \mathrm{C}$ ratio would not follow that of $\mathrm{CO}$, but be substantially higher if methanol is mainly formed by gas-phase chemistry in an environment where fractionation is active. Observations of the ${ }^{12} \mathrm{C} /{ }^{13} \mathrm{C}$ ratio in other molecules than CO towards our sources are scarce, but Henkel et al. (1983) measured a $\mathrm{H}_{2}^{12} \mathrm{CO} / \mathrm{H}_{2}^{13} \mathrm{CO}$ ratio of 66 towards DR21, which is somewhat higher than our values towards the same source $(55 \pm 8$ and $58 \pm 3$ for methanol and $\mathrm{C}^{18} \mathrm{O}$, respectively). This is indicative of ongoing fractionation since formaldehyde $\left(\mathrm{H}_{2} \mathrm{CO}\right)$ is part of the "carbon isotope pool" according to Langer et al. (1984). On the other hand, formaldehyde is also expected to be formed on grain surfaces, as a step in the $\mathrm{CO}$ hydrogenation process leading to methanol, and in that case get a similarly low ratio. The higher ratio observed in formaldehyde does not necessarily mean that the grain surface production does not take place, but could instead be an indication of a more efficient gas-phase production. It would be interesting to compare the ${ }^{12} \mathrm{C} /{ }^{13} \mathrm{C}$ ratio in methanol and formaldehyde towards more of our sources.

Savage et al. (2002) present observations of the ${ }^{12} \mathrm{C} /{ }^{13} \mathrm{C}$ ratio in $\mathrm{CN}$ towards a number of galactic molecular clouds, including DR21 (OH) and W51 M (less than 10" from our targeted position in W51). They report a ratio of $35 \pm 12$ in W51 M, which 
is higher than for methanol, $18 \pm 3$, but within the errorbars for what is measured by Langer \& Penzias (1990) for $\mathrm{C}^{18} \mathrm{O}, 46 \pm 2$. This comparison can be explained if the ${ }^{12} \mathrm{CH}_{3} \mathrm{OH}$ emission we observe has a high optical depth, as discussed in Sect. 4, decreasing the integrated intensity ratio as compared to the isotopic ratio. For DR21 (OH), Savage et al. (2002) derive a ${ }^{12} \mathrm{C} /{ }^{13} \mathrm{C}$ ratio from $\mathrm{CN}$ which is noticeably lower than our values, $36 \pm 3$ as compared to $55 \pm 8$ in methanol and $58 \pm 3$ in $\mathrm{C}^{18} \mathrm{O}$. Even though a ${ }^{13} \mathrm{C}$ fractionation process, similar to that in $\mathrm{CO}$ (Eq. (1)), has been predicted to occur in CN with a $\Delta E=34 \mathrm{~K}$ (Langer 1992), this does not account for such a small ratio as compared to $\mathrm{C}^{18} \mathrm{O}$. Doubly peaked line profiles towards DR21 $(\mathrm{OH})$ are reported to have complicated the analysis (Savage et al. 2002), and might be a source of error unaccounted for in the derived $\mathrm{CN}$ ratio.

It is not firmly established how the nuclear spin temperature is connected to the physical temperature at molecular formation. However, in most exoergic gas-phase reactions the excess energy of the reaction is very large compared to the mean kinetic energy in the gas, and large compared to the rotational energy splittings (except in light hydrides). It can therefore be assumed that the population distribution of molecules formed or altered by such a reaction is thermalized at a high temperature, in the case of methanol leading to an E/A ratio of one, which is not observed. Also, observations of the deuterated versions of methanol and formaldehyde in pre-stellar cores (Bacmann et al. 2007; Bergman et al. 2011), together with laboratory experiments of H-D substitutions in methanol on cold grain surfaces (Hidaka et al. 2009), seem to indicate that proton exchange reactions are more likely to take place on grain surfaces than in the gas-phase. In addition, the exchange seems to be with the hydrogen atoms of the methyl group rather than with that of the $\mathrm{OH}$ radical (Parise et al. 2004). Although not adding any information on the rates of proton exchange reactions in the gas-phase, this does argue that methanol molecules are released from the grains with an E/A ratio equilibrated at the temperature of the dust grain itself. That is, the spin temperature and formation temperature should be closely connected, at least initially.

Results of the E/A ratio test are inconclusive, for most sources being consistent with formation or equilibration in the gas-phase, but for two indicating low formation temperatures associated with formation on cold grain surfaces. The two sources, W51 e1/e2 and AFGL 2591, are not among those showing a correlation between ${ }^{12} \mathrm{C} /{ }^{13} \mathrm{C}$ ratios in methanol and $\mathrm{CO}$ (towards AFGL 2591 the methanol signals were too weak, see Sect. 2), but are deemed likely to have an emission contribution from thermally evaporated methanol close to their YSOs, see Sect. 3. However, to clarify whether an overabundance of A-methanol can be correlated with the desorption mechanism, or some aspect of the physical properties of the methanol gas, is beyond the scope of this study.

The RADEX model does not present unique solutions to the temperature, density and column density of the emitting gas, especially since it models a homogeneous medium within the beam while astronomical sources tend to have structure like gradients, clumps or filaments. We intend to use an enhanced version of the RADEX code to further model the excitation and formation of methanol molecules. This model will allow us to include an additional radiation field as experienced by the molecules, corresponding to the dust continuum radiation from the embedded YSO, as well as a possibility to control the overall $\mathrm{E} / \mathrm{A}$ ratio of the model via assumptions on formation and destruction. It will also incorporate new collision rates published by Rabli \& Flower (2010). This work will be presented in a forthcoming paper.

\section{Conclusions}

We show that the ${ }^{12} \mathrm{C} /{ }^{13} \mathrm{C}$ isotopic ratio is very similar in gasphase $\mathrm{CH}_{3} \mathrm{OH}$ and $\mathrm{C}^{18} \mathrm{O}$, on the spatial scale of about $40^{\prime \prime}$, towards four massive YSOs. All of these sources are at such an early stage of evolution that they have not yet evolved any substantial hot cores. Our interpretation is that the observed emission originates in the extended, cold envelopes of the YSOs, where the methanol abundance is maintained by non-thermal desorption from dust grains. The similarity in ${ }^{12} \mathrm{C} /{ }^{13} \mathrm{C}$ isotopic ratio between $\mathrm{CH}_{3} \mathrm{OH}$ and $\mathrm{CO}$ then poses an argument in favour of methanol formation from $\mathrm{CO}$ on the cold grain surfaces.

Methanol spin temperatures of 10 and $16 \mathrm{~K}$ are derived from the observed nuclear spin type ratios (E/A) towards two YSOs, while $T_{\text {spin }}>22 \mathrm{~K}$ are found for five others. The inconclusive results suggest that the E/A ratio test is not appropriate to use in these types of environments to distinguish between gas and grain surface formation of methanol.

We thus conducted two independent observational tests, one showing consistency with methanol formation from hydrogenation of $\mathrm{CO}$ on grain surfaces, and the other being inconclusive.

Acknowledgements. This work was supported in part by the Swedish Research Council (VR) and the Swedish National Space Board. In addition, E.S.W. was partially supported by NASA's Exobiology Program. Astrophysics at QUB is supported by a grant from the STFC. The authors appreciate constructive comments from the anonymous referee.

\section{Appendix A: Gas-phase reactions for interstellar methanol}

In Sect. 4.2, we assume that the E/A abundance ratios derived from the observations reflect the ice temperatures at which methanol form on grain surfaces. This is correct if proton exchange reactions occur on longer time-scales than other processes that destroy methanol. In molecular clouds the primary reactive partners that cause proton transfer are $\mathrm{H}^{+}, \mathrm{H}_{3}^{+}$and $\mathrm{HCO}^{+}$, with the latter two more important because of their larger abundances. The ions that destroy $\mathrm{CH}_{3} \mathrm{OH}$ without causing proton exchange include $\mathrm{C}^{+}$and $\mathrm{He}^{+}$.

The abundance of $\mathrm{H}_{3}^{+}$in molecular clouds can be estimated by balancing its formation and destruction processes, namely, cosmic ray ionization of $\mathrm{H}_{2}$ and reaction with $\mathrm{CO}$, respectively. Thus, $n\left(\mathrm{H}_{3}^{+}\right)=\zeta n\left(\mathrm{H}_{2}\right) / k n(\mathrm{CO})$, where $\zeta$ is the cosmic ray ionization rate and $k$ is the rate coefficient for proton transfer with $\mathrm{CO}$. With canonical values for these rates, $n\left(\mathrm{H}_{3}^{+}\right)=10^{-8} / f(\mathrm{CO})$, where $f(\mathrm{CO})$ is the fractional abundance of $\mathrm{CO}$ and is reasonably independent of temperature and density except in regions in which CO is depleted.

Although $\mathrm{H}_{3}^{+}$normally transfers a proton upon collision with a neutral species having a larger proton affinity than $\mathrm{H}_{2}$, the case of reaction with $\mathrm{CH}_{3} \mathrm{OH}$ is interestingly different. In this case the dominant channel, by a factor of four, is dissociative proton transfer (Lee et al. 1992):

$$
\mathrm{H}_{3}^{+}+\mathrm{CH}_{3} \mathrm{OH} \longrightarrow \mathrm{CH}_{3}^{+}+\mathrm{H}_{2} \mathrm{O}+\mathrm{H}_{2}
$$

which has a room temperature rate coefficient of $3.7 \times$ $10^{-9} \mathrm{~cm}^{3} \mathrm{~s}^{-1}$, and not proton transfer:

$\mathrm{H}_{3}^{+}+\mathrm{CH}_{3} \mathrm{OH} \longrightarrow \mathrm{CH}_{3} \mathrm{OH}_{2}^{+}+\mathrm{H}_{2}$.

At low temperatures these rate coefficients scale roughly as $T^{-0.5}$ due to ion-dipole effects. Thus at $15 \mathrm{~K}$, the time-scale for destruction of $\mathrm{CH}_{3} \mathrm{OH}$ by $\mathrm{H}_{3}^{+}$is roughly $\left(1.7 \times 10^{-8} n\left(\mathrm{H}_{3}^{+}\right)\right)^{-1} \mathrm{~s}$, or around $2 \times 1^{9} f(\mathrm{CO}) \mathrm{yr}$. 
E. S. Wirström et al.: Observational tests of interstellar methanol formation
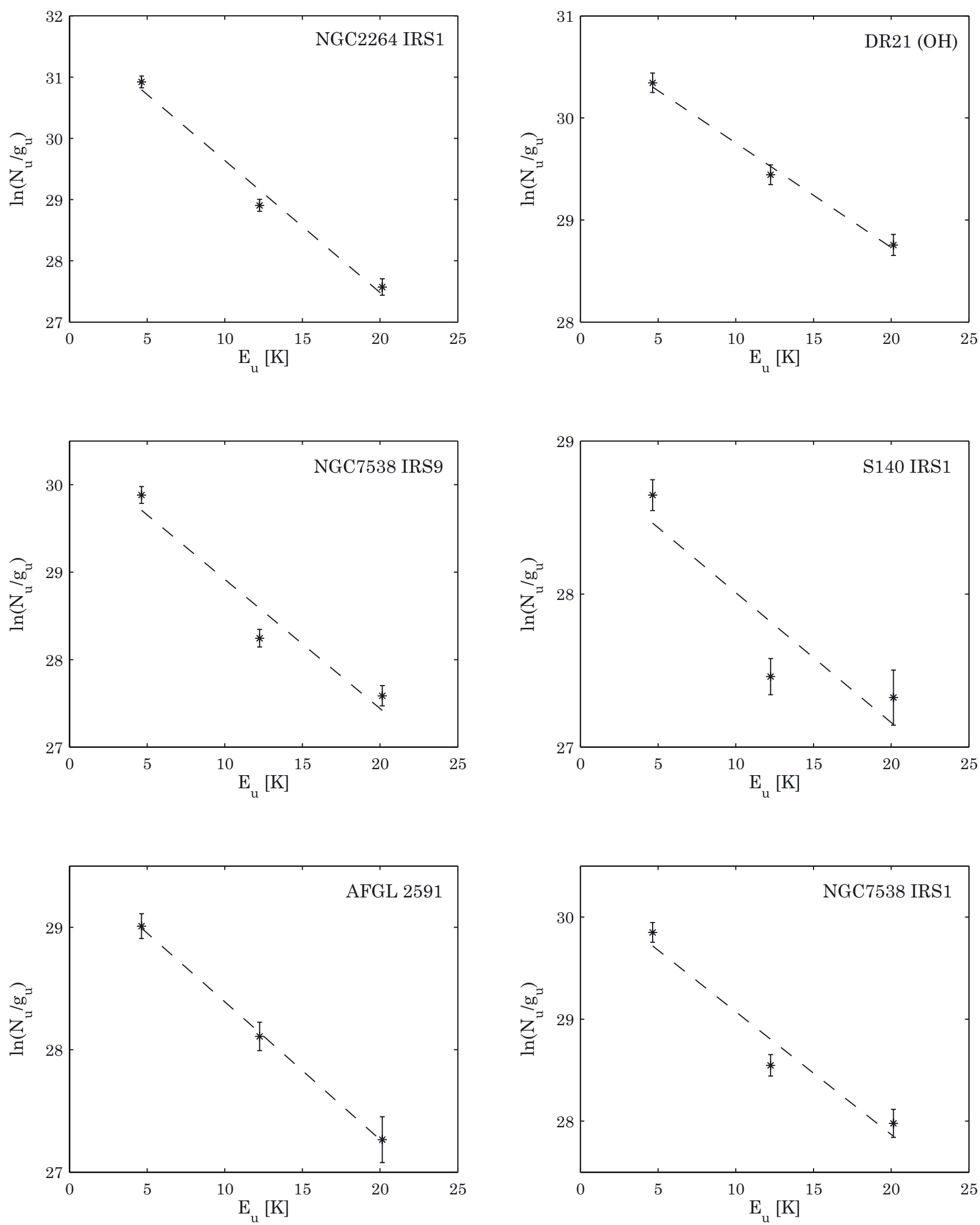

Fig. A.1. Rotational diagrams for the $\mathrm{E}-\mathrm{CH}_{3} \mathrm{OH}$ lines towards sources included in the $\mathrm{E}$ to A ratio analysis. The straight lines represent linear least square fits with slopes given by $-1 / T_{\text {rot }}$, see Table 4 for the corresponding $T_{\text {rot }}$. Error bars indicate observational uncertainties including a $10 \%$ systematic error. 
Table A.1. Gaussian line parameters.

\begin{tabular}{|c|c|c|c|c|c|c|}
\hline \multirow[b]{2}{*}{ Source } & & \multicolumn{3}{|l|}{${ }^{12} \mathrm{CH}_{3} \mathrm{OH}$} & \multicolumn{2}{|l|}{${ }^{13} \mathrm{CH}_{3} \mathrm{OH}$} \\
\hline & $\begin{array}{c}v_{\mathrm{c}} \\
(\mathrm{MHz})\end{array}$ & $\begin{array}{c}\text { Ampl. } \\
(\mathrm{K})\end{array}$ & $\begin{array}{c}\Delta v \\
\left(\mathrm{~km} \mathrm{~s}^{-1}\right)\end{array}$ & $\begin{array}{c}v_{\mathrm{c}} \\
(\mathrm{MHz})\end{array}$ & $\begin{array}{c}\text { Ampl. } \\
(\mathrm{mK})\end{array}$ & $\begin{array}{c}\Delta v \\
\left(\mathrm{~km} \mathrm{~s}^{-1}\right)\end{array}$ \\
\hline \multirow[t]{4}{*}{ W51 e1/e2 } & $96739.990(61)^{a}$ & $3.76(5)$ & $8.91(21)$ & $94405.35(9)$ & $169(10)$ & $6.4(3)$ \\
\hline & $96742.107(38)^{a}$ & $3.21(12)$ & $6.17(18)$ & $94407.47(8)$ & $243(6)$ & $7.6(4)$ \\
\hline & $96744.944(23)^{a}$ & $2.03(23)$ & $7.46(16)$ & $94411.28(3)$ & $131(3)$ & $7.1(2)$ \\
\hline & $96755.877(31)^{a}$ & $1.25(2)$ & $7.30(15)$ & $94420.68(4)$ & $95(3)$ & $6.9(3)$ \\
\hline \multirow[t]{4}{*}{ NGC 2264 IRS1 } & $96739.516(3)$ & $3.49(1)$ & $5.11(2)$ & $94405.43(6)^{a}$ & $80(8)$ & $3.4(5)$ \\
\hline & $96741.525(2)$ & $4.58(1)$ & $3.48(1)$ & $94407.30(7)^{a}$ & $81(8)$ & $3.6(5)$ \\
\hline & $96744.602(9)$ & $0.762(10)$ & $4.14(7)$ & $94410.90(8)^{a}$ & $39(17)$ & $0.8(4)$ \\
\hline & $96755.581(30)$ & $0.205(12)$ & $3.14(25)$ & & $\ldots$ & \\
\hline \multirow[t]{4}{*}{ DR21 (OH) } & $96739.454(5)$ & $1.96(1)$ & $5.10(5)$ & $94405.43(4)^{a}$ & $32(1)$ & $6.3(4)$ \\
\hline & $96741.485(4)$ & $2.84(1)$ & $4.21(3)$ & $94407.33(2)^{a}$ & $56(2)$ & $3.7(2)$ \\
\hline & $96744.514(10)$ & $1.04(1)$ & $5.21(7)$ & $94411.05(25)^{a}$ & $9(1)$ & $8.8(2.8)$ \\
\hline & $96755.463(22)$ & $0.42(1)$ & $5.04(16)$ & $\ldots$ & $\ldots$ & $\ldots$ \\
\hline \multirow[t]{4}{*}{ NGC 7538 IRS9 } & $96739.462(6)$ & $1.31(1)$ & $4.83(4)$ & $94406.92(20)^{a}$ & $26(2)$ & $6.6(1.2)$ \\
\hline & $96741.420(10)$ & $1.81(1)$ & $3.98(6)$ & $94405.22(11)^{a}$ & $12(4)$ & $4.6(1.8)$ \\
\hline & $96744.538(16)$ & $0.380(7)$ & $4.30(12)$ & $\ldots$ & $\ldots$ & $\ldots$ \\
\hline & $96755.540(33)$ & $0.143(6)$ & $4.52(25)$ & $\ldots$ & $\ldots$ & . \\
\hline \multirow[t]{4}{*}{ S140 IRS1 } & $96739.390(11)$ & $0.649(16)$ & $2.82(8)$ & $94407.04(1)^{a}$ & $34(7)$ & $0.4(1)$ \\
\hline & $96741.396(13)$ & $1.02(2)$ & $2.40(5)$ & $94405.20(2)^{a}$ & $34(8)$ & $0.3(1)$ \\
\hline & $96744.628(20)$ & $0.359(18)$ & $2.08(12)$ & $94410.92(2)^{a}$ & $19(9)$ & $0.3(1)$ \\
\hline & $96755.623(52)$ & $0.155(15)$ & $3.26(43)$ & $\ldots$ & $\ldots$ & $\ldots$ \\
\hline \multirow[t]{4}{*}{ NGC 7538 IRS1 } & $96739.456(9)$ & $1.37(2)$ & $4.45(7)$ & $\ldots$ & $\ldots$ & $\ldots$ \\
\hline & $96741.448(9)$ & $1.96(2)$ & $3.87(5)$ & $\ldots$ & $\ldots$ & $\ldots$ \\
\hline & $96744.606(21)$ & $0.555(16)$ & $3.97(14)$ & $\ldots$ & $\ldots$ & $\ldots$ \\
\hline & $96755.638(35)$ & $0.291(18)$ & $3.32(28)$ & $\ldots$ & $\ldots$ & $\ldots$ \\
\hline \multirow[t]{4}{*}{ AFGL 2591} & $96739.376(14)$ & $0.775(17)$ & $3.39(10)$ & $\ldots$ & $\ldots$ & $\ldots$ \\
\hline & $96741.454(15)$ & $1.06(1)$ & $5.42(10)$ & $\ldots$ & $\ldots$ & $\ldots$ \\
\hline & $96744.523(30)$ & $0.38(2)$ & $3.71(0.21)$ & $\ldots$ & $\ldots$ & $\ldots$ \\
\hline & $96755.585(50)$ & $0.170(19)$ & $2.80(36)$ & $\ldots$ & $\ldots$ & $\ldots$ \\
\hline
\end{tabular}

Notes. Gaussians fitted to spectra on the $T_{\mathrm{mb}}$ scale, where $v_{\mathrm{c}}$ is the Gaussian central frequency. The formal $1 \sigma$ error on the last digit of each parameter is given in parentheses. No entry indicates that either no spectrum is available, or no Gaussian could be fitted. ${ }^{(a)}$ Fit not used in any of the analysis.

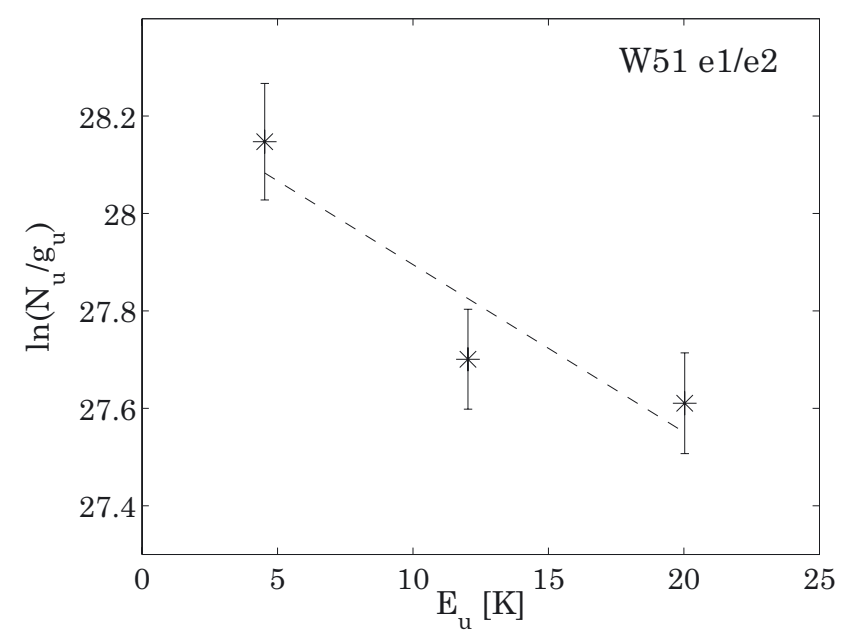

Fig. A.2. Rotational diagram for the $\mathrm{E}^{-13} \mathrm{CH}_{3} \mathrm{OH}$ lines towards $\mathrm{W} 51$ e1/e2. The straight line represents the linear least square fit to the data with a slope given by $-1 / T_{\text {rot }}$, see Table 4 for the corresponding $T_{\text {rot }}$. Error bars indicate observational uncertainties including a $10 \%$ systematic error.

The proton exchange time-scale depends on the rate coefficient for the proton transfer of $\mathrm{HCO}^{+}$with $\mathrm{CH}_{3} \mathrm{OH}-$ some $25 \%$ smaller than that for dissociative proton transfer with $\mathrm{H}_{3}^{+}$
- and the abundance of $\mathrm{HCO}^{+}$compared to those ions that destroy methanol, $\mathrm{H}_{3}^{+}, \mathrm{C}^{+}$and $\mathrm{He}^{+}$. Model calculations indicate that these ions can have abundances comparable to or greater than that of $\mathrm{HCO}^{+}$, and particularly in clouds in which $\mathrm{CO}$ is depleted where $\mathrm{He}^{+}$and the deuterated analogues of $\mathrm{H}_{3}^{+}-$which also destroy methanol - can have very large abundances relative to $\mathrm{HCO}^{+}$.

From these order of magnitude estimates, it appears that methanol is destroyed relatively rapidly in molecular clouds and before the E/A ratios can be reset from those determined by its formation on interstellar ices, although this conclusion rests on estimates of ion abundances that are not easily constrained by observation.

\section{References}

Acharyya, K., Fuchs, G. W., Fraser, H. J., van Dishoeck, E. F., \& Linnartz, H. 2007, A\&A, 466, 1005

Allamandola, L. J., Sandford, S. A., Tielens, A. G. G. M., \& Herbst, T. M. 1992, ApJ, 399, 134

Amiaud, L., Dulieu, F., Fillion, J., Momeni, A., \& Lemaire, J. L. 2007, J. Chem. Phys., 127, 144709

Araya, E. D., Kurtz, S., Hofner, P., \& Linz, H. 2009, ApJ, 698, 1321

Bachiller, R., Codella, C., Colomer, F., Liechti, S., \& Walmsley, C. M. 1998, A\&A, 335, 266

Bacmann, A., Lefloch, B., Parise, B., Ceccarelli, C., \& Steinacker, J. 2007, in Molecules in Space and Laboratory

Barbosa, C. L., Blum, R. D., Conti, P. S., Damineli, A., \& Figuerêdo, E. 2008, ApJ, 678, L55

Bergman, P., Parise, B., Liseau, R., \& Larsson, B. 2011, A\&A, 527, A39 
Boogert, A. C. A., Ehrenfreund, P., Gerakines, P. A., et al. 2000, A\&A, 353, 349 Boogert, A. C. A., Blake, G. A., \& Tielens, A. G. G. M. 2002, ApJ, 577, 271 Bottinelli, S., Adwin Boogert, A. C., Bouwman, J., et al. 2010, ApJ, 718, 1100 Brooke, T. Y., Sellgren, K., \& Geballe, T. R. 1999, ApJ, 517, 883

Chandler, C. J., Gear, W. K., \& Chini, R. 1993, MNRAS, 260, 337

Charnley, S. B., Ehrenfreund, P., Millar, T. J., et al. 2004, MNRAS, 347, 157

Crampton, D., \& Fisher, W. A. 1974, Publications of the Dominion Astrophysical Observatory Victoria, 14, 283

Crovisier, J. 2007, in Proc. 18th Rencontres de Blois: Planetary Science: Challenges and Discoveries, 28th May-2nd June 2006, Blois, France [arXiv: astro-ph/0703785]

Dartois, E., Schutte, W., Geballe, T. R., et al. 1999, A\&A, 342, L32

Friberg, P., Hjalmarson, A., Madden, S. C., \& Irvine, W. M. 1988, A\&A, 195, 281

Fuchs, G. W., Cuppen, H. M., Ioppolo, S., et al. 2009, A\&A, 505, 629

Garrod, R. T., Park, I. H., Caselli, P., \& Herbst, E. 2006, Faraday Discussions, 133,51

Garrod, R. T., Wakelam, V., \& Herbst, E. 2007, A\&A, 467, 1103

Genzel, R., Downes, D., Schneps, M. H., et al. 1981, ApJ, 247, 1039

Geppert, W. D., Hamberg, M., Thomas, R. D., et al. 2006, Faraday Discussions, 133,177

Gibb, E. L., Whittet, D. C. B., Boogert, A. C. A., \& Tielens, A. G. G. M. 2004 , ApJS, 151, 35

Henkel, C., Wilson, T. L., Walmsley, C. M., \& Pauls, T. 1983, A\&A, 127, 388

Hidaka, H., Watanabe, M., Kouchi, A., \& Watanabe, N. 2009, ApJ, 702, 291

Ioppolo, S., Palumbo, M. E., Baratta, G. A., \& Mennella, V. 2009, A\&A, 493, 1017

Kawakita, H., \& Kobayashi, H. 2009, ApJ, 693, 388

Kawakita, H., Dello Russo, N., Furusho, R., et al. 2006, ApJ, 643, 1337

Langer, W. D. 1992, in Astrochemistry of Cosmic Phenomena, ed. P. D. Singh, IAU Symp., 150, 193

Langer, W. D., \& Penzias, A. A. 1990, ApJ, 357, 477

Langer, W. D., Graedel, T. E., Frerking, M. A., \& Armentrout, P. B. 1984, ApJ, 277,581

Lee, H. S., Drucker, M., \& Adams, N. G. 1992, Int. J. Mass Spectr. Ion Proc., 117,101
Liszt, H. S., Pety, J., \& Lucas, R. 2008, A\&A, 486, 493

Mekhtiev, M. A., Godfrey, P. D., \& Hougen, J. T. 1999, J. Mol. Spect., 194, 171 Menten, K. M., Walmsley, C. M., Henkel, C., \& Wilson, T. L. 1988, A\&A, 198, 253

Moscadelli, L., Reid, M. J., Menten, K. M., et al. 2009, ApJ, 693, 406

Müller, H. S. P., Schlöder, F., Stutzki, J., \& Winnewisser, G. 2005, J. Mol. Struct., 742,215

Neri, L. J., Chavarria-K., C., \& de Lara, E. 1993, A\&AS, 102, 201

Öberg, K. I., van Dishoeck, E. F., \& Linnartz, H. 2009, A\&A, 496, 281

Pardanaud, C., Crovisier, J., Bockelée-Morvan, D., \& Biver, N. 2007, in Molecules in Space and Laboratory

Parise, B., Castets, A., Herbst, E., et al. 2004, A\&A, 416, 159

Pipenbrink, A., \& Wendker, H. J. 1988, A\&A, 191, 313

Pontoppidan, K. M., Dartois, E., van Dishoeck, E. F., Thi, W.-F., \& d'Hendecourt, L. 2003, A\&A, 404, L17

Pontoppidan, K. M., Boogert, A. C. A., Fraser, H. J., et al. 2008, ApJ, 678, 1005 Pottage, J. T., Flower, D. R., \& Davis, S. L. 2004, MNRAS, 352, 39

Rabli, D., \& Flower, D. R. 2010, MNRAS, 406, 95

Remijan, A., Sutton, E. C., Snyder, L. E., et al. 2004, ApJ, 606, 917

Roser, J. E., Vidali, G., Manicò, G., \& Pirronello, V. 2001, ApJ, 555, L61

Sandell, G., Goss, W. M., Wright, M., \& Corder, S. 2009, ApJ, 699, L31

Savage, C., Apponi, A. J., Ziurys, L. M., \& Wyckoff, S. 2002, ApJ, 578, 211

Shen, C. J., Greenberg, J. M., Schutte, W. A., \& van Dishoeck, E. F. 2004, A\&A, 415, 203

Turner, B. E. 1998, ApJ, 501, 731

Ulich, B. L., \& Haas, R. W. 1976, ApJS, 30, 247

van der Tak, F. F. S., van Dishoeck, E. F., Evans, II, N. J., Bakker, E. J., \& Blake, G. A. 1999, ApJ, 522, 991

van der Tak, F. F. S., van Dishoeck, E. F., \& Caselli, P. 2000, A\&A, 361, 327

van der Tak, F. F. S., Black, J. H., Schöier, F. L., Jansen, D. J., \& van Dishoeck, E. F. 2007, A\&A, 468, 627

Watanabe, N., Nagaoka, A., Shiraki, T., \& Kouchi, A. 2004, ApJ, 616, 638

Willacy, K., \& Millar, T. J. 1998, MNRAS, 298, 562

Young, E. D., \& Schauble, E. A. 2011, in 42nd Lunar and Planetary Science Conference 